\title{
The suffix effect: Postcategorical attributes in a serial recall paradigm
}

\author{
ROCHELLE L. HARRIS, JOHN GAUSEPOHL, ROBIN J. LEWIS \\ and KATHRYN T. SPOEHR \\ Brown University, Providence, Rhode Island 02912
}

\begin{abstract}
A serial list learning paradigm was employed to investigate the role of postcategorical information in producing the stimulus suffix effect (SSE). Serial recall performance was measured for eight-item word lists under four experimental conditions: a control condition (C), where white noise was used as a stimulus suffix; the word-suffix (WS) condition, where list items and suffix were semantically unrelated; the target-suffix condition (TS), in which the final list item and the suffix were from the same category; and the category-suffix condition, in which the final three list elements and the suffix were from the same category. All three verbal suffix conditions produced the SSE, but its magnitude was reduced when the suffix was semantically related to the last list element. Several pre- and postcategorical explanations of the SSE are evaluated in light of the results.
\end{abstract}

In serial recall tasks, if list items are presented auditorially, the final items in the list are better recalled than if the list is presented visually. This advantage of auditory presentation has been labeled the modality effect, and has been attributed to the contribution of an additional auditory memory store (Crowder \& Morton, 1969). The auditory advantage disappears, however, if the recall list is followed by an extra item, even when recall of that item is not required. This decrement in recall performance, called the "stimulus suffix effect" (SSE), can be attributed to the negative effect of the irrelevant item on the information in the auditory store that would otherwise produce the modality effect.

One recent version of the auditory store explanation assumes that the additional memory component responsible for the advantage of auditory presentation is organized precategorically (Crowder, 1978). In this context, categorical refers to the point in information processing at which contact is first made between new input and learned linguistic categories. The precategorical model proposes that the auditory memory of this echoic component is functionally uncorrelated with the whole semantic or categorical memory system, and resides in a separate store called precategorical acoustic storage (PAS) (Crowder \& Morton, 1969). Stimulus information in the PAS is carried as raw auditory sensory energy and is subject to only primitive processing. For categorization to occur, a further stage of information processing that compares the auditory memory in PAS with the learned linguistic information is necessary. According to the precategorical model, the SSE is the result of displacement of the precategorical acoustic traces by the incoming information at a first stage of processing.

A second hypothesis concerning the manner of organization in this echoic component assumes that information storage is postcategorical. The postcategorical model, discussed by Salter and Colley (1977), proposes that simultaneous to its storage at a sensory level, auditory information is registered against its permanent categorical memory representation. This categorical processing is explained as excitation of distinct "locations" in a semantically organized lexical memory (Meyer, Schvaneveldt, \& Ruddy, 1975). When information is assessed at a particular memory location, excitation spreads to other nearby locations. According to a postcategorical model, the SSE is not simply a result of displacement of raw sensory energy. Instead, the suffix displaces some sensory information and acts to block more extensive coding and rehearsal processes, which facilitate recall.

Salter and Colley (1977) proposed the postcategorical model to explain the results of an experiment in which changing the postcategorical attributes of a verbal stimulus suffix affected the last item recalled. In their study, stimulus lists were followed by one of three types of suffixes: a word suffix unrelated to the other words in the stimulus list; a word suffix synonymous to the last, or target, item of the stimulus list; or a nonverbal control suffix, a burst of white noise. It was found that both word suffixes produced a decrement in recall performance compared to the control, but that the interference due to a word suffix was significantly less when the target item and suffix were synonyms than when the two were semantically unrelated. They suggested that the synonym suffix provided dual excitation to the memory location of the target item, thereby enhancing recall and reducing the performance decrement caused by a word suffix.

The present experiment further investigated the consequences of varying the postcategorical attributes 
of a word suffix in a serial recall task. The verbal suffixes in the three experimental conditions differed in terms of their semantic relatedness to the recall items. In one condition, the suffix was unrelated to the recall items. In the other experimental conditions, the suffix was semantically related to either the final, target, item or to the final three recall items. The specific semantic relation was one of belonging to a superordinate category; "peach, grape, and pear," for example, are semantically related by virtue of being a "type of fruit," whereas "tree, doll, and steel" have no such relation to one another. In the control condition, the suffix consisted of a burst of white noise; previous research established that recall of a final item is no worse with the addition of white noise than with no suffix presented at all (Crowder \& Morton, 1969).

A suffix effect was anticipated for all three experimental conditions, but its magnitude should decrease significantly when the target word and suffix are of the same superordinate category. If the lexical memory model proposed by Salter and Colley (1977) is correct, then the eighth word of a list with the final three recall items of the same superordinate should have significantly fewer errors than a list with only the eighth item and the stimulus suffix of the same category. This is because the eighth item of the former list is excited four times compared to dual excitation of the latter list. Also, if only secondary excitation is required to enhance recall, then a decrease in errors should occur for Serial Positions 6 and 7, as well as Position 8, when these items are of the same superordinate category as the suffix.

\section{METHOD}

\section{Subjects}

Forty-eight undergraduates served as subjects; all were unpaid volunteers from Brown University. Twelve subjects were randomly assigned to each of four suffix groups. Subjects were tested in groups of five or seven at a time.

\section{Materials and Design}

Stimulus lists were constructed from a pool of 180 words. Eighty of the words were taken from 20 category lists of nouns or adjectives selected at random from Battig and Montague (1969). From each of the 20 selected lists, the four most frequent monosyllabic responses were chosen for the word pool. The additional 100 words, also monosyllabic nouns or adjectives, were randomly chosen from Thorndike and Lorge (1931). These words were matched in frequency ratings to those of the category lists, but were otherwise unrelated.

Twenty lists of each of four different types were constructed. The control lists (C) consisted of eight randomly chosen words, with the constraint that no two were from the same category. In the word-suffix lists (WS), the eight base words plus the suffix word were randomly chosen with the same constraint. In both the target-suffix (TS) and category-suffix lists (CS), the words were generated in the same fashion up to the critical list position. For the TS lists, the target word (Serial Position 8) and the suffix word were the two most frequent responses in a particular category. In the CS lists, the final three words (Serial Positions 6, 7, and 8) and the suffix words were from the same category, and all four were of approximately the same response frequency.

\section{Procedure}

All list items and suffixes were tape-recorded in a female voice at a rate of one item per $.6 \mathrm{sec}$. The interstimulus interval between the target word and the suffix word was the same as that between all other adjacent list words. To distinguish the suffix word from the recall list, subjects were instructed to attend to a white light, which was turned on throughout the presentation of the recall list but switched off immediately prior to the presentation of the suffix. The light also served as a "ready" signal; it went on $1 \mathrm{sec}$ before each trial began.

On each trial, the subject heard a list of eight items followed by the suffix, which was either a word or a burst of white noise. After presentation of each list, subjects were allowed approximately $20 \mathrm{sec}$ for written recall in the prepared response booklets. On each page of the booklet, a vertical row of eight lines was printed, one line per serial position in the list. Prior to the onset of the experiment, it was pointed out to subjects in the WS, TS, and CS conditions that the suffix was not part of the recall list. Instructions for the recall procedure specified that responses were to be made from the beginning of the list onward, in serial order, without backtracking. Subjects were told that an item was correct only if it appeared in the proper order, and they were encouraged to guess rather than leave a line blank.

During three initial practice trials, subjects were carefully watched to insure that the procedure was being carried out as instructed. Subjects then proceeded through 20 lists for their condition.

\section{RESULTS}

Any response that did not match the stimulus word in a particular serial position was scored as an error. The total number of recall errors per serial position across all 20 trials was tallied for each subject and then averaged for each of the four experimental conditions; each mean was based upon 240 observations. Figure 1 shows these errors plotted as a function of serial position for each condition.

A two-way analysis of variance showed a significant main effect of serial position $[F(7,77)=140.7$, MSe $=210.3, \quad p<.001], \quad a$ main effect of suffix

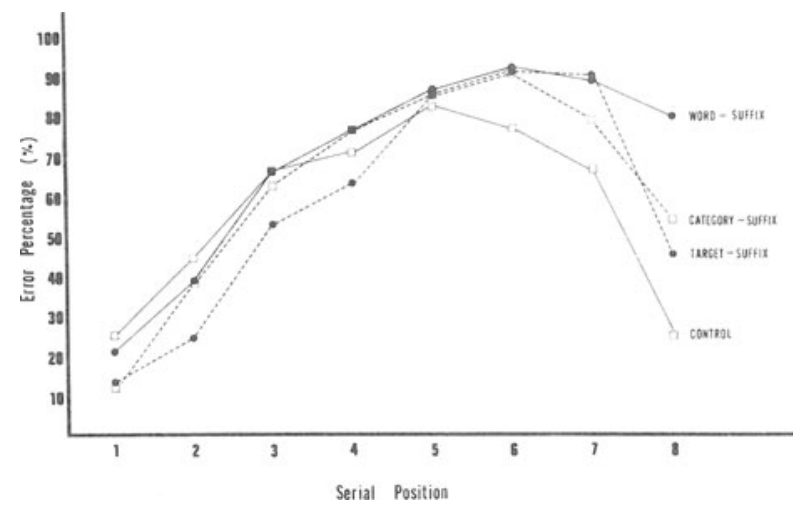

Figure 1. Percent errors as a function of serial position for the control (C), word-suffix (WS), target-suffix (TS), and category-suffix (CS) conditions. 
condition $[\mathrm{F}(3,33)=3.70, \mathrm{MSe}=696.9, \mathrm{p}<.05]$, and a significant interaction of Serial Position by Suffix Condition $[\mathrm{F}(21,231)=6.37, \mathrm{MSe}=177.1, \mathrm{p}<.001]$. A Newman-Keuls procedure located the source of the interaction, and thus possibly the source of the main effect of suffix condition, at Serial Position 8 . There were no statistically significant differences between the curves except at Position 8, where WS was significantly higher $(\mathrm{p}<.01)$ than any of the other conditions and CS was significantly above the control. No significant difference was found between the TS and CS conditions at this position. The CS condition did, however, show a modest recency effect at Position 7, whereas the other experimental conditions did not. Although the recency effect did not reach statistical significance, it does suggest a higher probability of recall for Condition CS at that position.

\section{DISCUSSION}

As the results of this experiment demonstrate, recall for the last item is differentially affected by specific attributes of the suffix item. A suffix effect was found in all the verbal suffix conditions, for recall of the target items was significantly worse with a word suffix than it was when the suffix was a burst of white noise. The performance decrement caused by a verbal suffix was diminished, however, when the word suffix was semantically related to either the last three or the final items in the recall list.

A precategorical model of auditory memory could adequately account for the difference in performance between a noise suffix and a verbal suffix. Crowder (1978) pointed out several types of classification possible on a precategorical basis, including discrimination of voices, of different spatial locations, or of intensity dynamics. However, an explanation of the suffix effect based solely upon precategorical information does not explain the significantly better recall of the target item in the present experiment, since the suffixes of interest here varied only on postcategorical factors.

It could be argued that the improved recall was due to an awareness of semantic relatedness between the target and suffix. Such an explanation, called the redirected attention hypothesis (Salter \& Colley, 1977), proposes that the meaningful association between the list word and suffix causes a diversion of attention to the final list items at the expense of earlier items. One could thus explain the lessened effect of the suffix due to an increase in attention to the target item. In our data, however, there is no evidence of a diversion of attention. Items in Serial Positions 1-7 were recalled equally well in all the verbal suffix conditions, including the category-suffix condition, where one might expect an awareness of semantic relatedness.

Salter and Colley (1977) proposed that the recall probability of a target word results from two separate factors: extrinsic interference with the acoustic attributes of the target word and any intrinsic facilitation that would result if the target and suffix were meaningfully associated. In the present experiment, extrinsic interference was equivalent across the three verbal suffix conditions. Thus, the significantly higher recall for the two conditions in which the target and suffix were related suggests intrinsic facilitation. However, this explanation is weakened when the underlying lexical memory model of Meyer et al. (1975) is examined. In this model, facilitation occurs when residual neural activity produced from information assessment in one memory location spreads to other nearby locations. Salter and Colley (1977) contend that it is this dual excitation of the lexical memory location that enhances recall. Two of our findings are inconsistent with this explanation. First, the model predicts significantly better recall for Position 8 of the CS condition than for Position 8 of the TS condition. This prediction is derived from the likelihood that the eighth item receives more excitation in the CS than in the TS condition. Also, if the requirement for enhanced recall is simply multiple excitation, then the words in Positions 6 and 7 of the CS condition should have exhibited fewer recall errors, since these items also experienced multiple excitation. Our data, however, display neither of these results.

A more accurate proposal would incorporate the notion of a threshold that would have to be achieved before a word could be successfully recalled. The Collins and Loftus (1975) memory model stipulates that activation from different sources sums and that, when the summation at a point of intersection reaches threshold, a response can be made. The results of our experiment are consistent with such a hypothesis. The lack of significantly fewer recall errors for Serial Position 8 in the CS condition as compared to the TS condition is suggestive of an all-or-none threshold. Moreover, the lack of enhanced recall for Serial Positions 6 and 7 of the CS condition indicates that the greater retention interval allows so much decay of activation that these items no longer exceed threshold at recall. The modest recency effect found in Position 7 of the CS condition also supports the threshold contention. Apparently, the threshold level was achieved by some subjects at this position, but, since the organization of the lexical memory system is variable with each individual, threshold was not reached by enough subjects to warrant a statistically significant decrease in recall errors.

\section{REFERENCES}

Battig, W. F., \& Montague, W. E. Categorizing norms for verbal items in 56 categories: A replication and extension of the Connecticut category norms. Journal of Experimental Psychology, 1969, 80, 1-46.

Collins, A. M., \& Loftus, E. F. A spreading activation theory of semantic processing. Psychological Review, 1975, 82, 407-428.

CROwDER, R. G. Memory for phonologically uniform lists. Journal of Verbal Learning and Verbal Behavior, 1978, 17, 73-89.

Crowder, R. G., \& Morton, J. Precategorical acoustic store. Perception \& Psychophysics, 1969, 5, 365-373.

Meyer, D. E., Schvaneveldt, R. W., \& Ruddy, M. G. Loci of contextual effects on visual word-recognition. In P. M. A. Rabbitt \& S. Dornic (Eds.), Attention and Performance $V$. London: Academic Press, 1975.

Salter, D., \& Colley, J. G. The stimulus suffix: A paradoxical effect. Memory \& Cognition, 1977, 5, 257-262.

THORNDIKE, E. L., \& LORGE, I. The teachers word book of 20,000 words. New York: Teachers College Press, Columbia University, 1931.

(Received for publication September 28, 1978.) 